\title{
Strain mapping in single-layer two-dimensional crystals via Raman activity
}

\author{
M. Yagmurcukardes, ${ }^{1}$ C. Bacaksiz, ${ }^{1}$ E. Unsal, ${ }^{2}$ B. Akbali, ${ }^{2}$ R. T. Senger,,${ }^{2,3}$ and H. Sahin ${ }^{3,4}$ \\ ${ }^{1}$ Department of Physics, University of Antwerp, Groenenborgerlaan 171, Antwerp B-2020, Belgium \\ ${ }^{2}$ Department of Physics, Izmir Institute of Technology, Izmir 35430, Turkey \\ ${ }^{3}$ ICTP-ECAR Eurasian Center for Advanced Research, Izmir Institute of Technology, Izmir 35430, Turkey \\ ${ }^{4}$ Department of Photonics, Izmir Institute of Technology, Izmir 35430, Turkey
}

(Received 28 December 2017; published 19 March 2018)

\begin{abstract}
By performing density functional theory-based $a b$ initio calculations, Raman-active phonon modes of singlelayer two-dimensional (2D) materials and the effect of in-plane biaxial strain on the peak frequencies and corresponding activities of the Raman-active modes are calculated. Our findings confirm the Raman spectrum of the unstrained 2D crystals and provide expected variations in the Raman-active modes of the crystals under in-plane biaxial strain. The results are summarized as follows: (i) frequencies of the phonon modes soften (harden) under applied tensile (compressive) strains; (ii) the response of the Raman activities to applied strain for the in-plane and out-of-plane vibrational modes have opposite trends, thus, the built-in strains in the materials can be monitored by tracking the relative activities of those modes; (iii) in particular, the $A$ peak in single-layer Si and Ge disappears under a critical tensile strain; (iv) especially in mono- and diatomic single layers, the shift of the peak frequencies is a stronger indication of the strain rather than the change in Raman activities; (v) Raman-active modes of single-layer $\operatorname{Re} X_{2}(X=\mathrm{S}, \mathrm{Se})$ are almost irresponsive to the applied strain. Strain-induced modifications in the Raman spectrum of 2D materials in terms of the peak positions and the relative Raman activities of the modes could be a convenient tool for characterization.
\end{abstract}

DOI: 10.1103/PhysRevB.97.115427

\section{INTRODUCTION}

The successful synthesis of graphene [1] opened a famous field of research, two-dimensional (2D) single-layer materials. 2D materials have attracted great attention due to their extraordinary electronic, optical, and mechanical properties that suit technological applications such as energy conversion, flexible electronics, and information technologies [2-5]. Following graphene, 2D single-layer structures such as transition-metal dichalcogenides (TMDs) [6-12], monoelemental 2D materials such as silicene [13,14], germanene [14], and groupIII-V binary compounds ( $h$-BN, $h$-AlN) [15-19] were successfully synthesized. In addition to those in-plane isotropic single layers, 2D materials with in-plane anisotropy such as $\mathrm{ReS}_{2}$ [10,20-22], $\mathrm{ReSe}_{2}$ [23,24], and black phosphorus (bp) were also widely studied [25-27]. The improved production methods, such as chemical vapor deposition and mechanical exfoliation, enable the synthesis of thinner and cleaner structures.

One of the most common techniques for the characterization of a material is Raman spectroscopy [28] which crops information about the nature of the material medium entities by monitoring the characteristic vibrational energy levels of the structure. It also provides nondestructive analysis and requires minimum sample preparation. In addition, Raman measurements are also able to provide information about the substratefree layer-number identification of layered materials [29-31], the strength of the interlayer coupling in layered materials [32], and interface coupling in van der Waals heterostructures $[33,34]$. Moreover, relative intensities of the Raman peaks lead to the determination of different-phase distributions in a material [35-38].
Strain can alter electronic and vibrational properties of materials $[39,40]$. Raman peak positions and intensities strongly depend on the presence of strain [41,42] since it modifies the crystal phonons, with stretching usually resulting in mode softening, and the opposite for compressing. The rate of change is summarized in the Gruneissen parameters which determines the thermomechanical properties. Moreover, strain enhances the Raman spectrum of 2D materials in terms of the Raman intensities. Although Raman spectroscopy has been widely studied in literature [43-45], detailed theoretical investigation of the strain effects on Raman peaks for 2D materials is still limited. Here, we theoretically investigate the strain-dependent vibrational properties of several 2D single-layer materials in terms of the peak frequencies and corresponding Raman activities.

The paper is organized as follows: Details of the computational methodology are given in Sec. II. The theory of Raman activity calculations is briefly explained in Sec. III. The evolution of peak frequencies and corresponding Raman activities under in-plane biaxial strain are discussed In Secs. III A-IIIC for monoatomic, diatomic, and isotropic single-layer TMDs, respectively, and those for in-plane anisotropic single layers of $\operatorname{Re} X_{2}$ and bp are presented in Secs. IIID 1 and IIID 2. Finally, we conclude in Sec. IV.

\section{COMPUTATIONAL METHODOLOGY}

For structural optimization and vibrational properties of considered materials, first-principle calculations were performed in the framework of density functional theory (DFT) as implemented in the Vienna ab initio Simulation Package 
(VASP) [46,47]. The Perdew-Burke-Ernzerhof (PBE) [48] form of generalized gradient approximation (GGA) was adopted to describe electron exchange and correlation. The van der Waals (vdW) correction was included by using the DFT-D2 method of Grimme [49]. The charge transfer between individual atoms was determined by the Bader technique [50].

The kinetic energy cutoff for plane-wave expansion was set to $800 \mathrm{eV}$ and the energy was minimized until its variation in the following steps became $10^{-8} \mathrm{eV}$. The Gaussian smearing method was employed for the total-energy calculations and the width of the smearing was chosen as $0.05 \mathrm{eV}$. Total HellmannFeynman forces in the until was reduced to $10^{-7} \mathrm{eV} / \AA$ for the structural optimization. $18 \times 18 \times 1 \Gamma$ centered $k$-point samplings were used or the primitive unit cells. For $2 \times 2$ reconstructed supercell of $\operatorname{Re} X_{2}$ structures, $k$-point sampling is reduced to $12 \times 12 \times 1$ mesh. To avoid interaction between the adjacent layers, our calculations were implemented with a vacuum space of $25 \AA$.

The vibrational properties of all single-layer crystals were calculated in terms of the off-resonant Raman activities of phonon modes at the $\Gamma$ point. For this purpose, first the vibrational phonon modes at the $\Gamma$ point were calculated using the finite-difference method as implemented in VASP. Each atom in the primitive unit cell was initially distorted $0.01 \AA$ and the corresponding dynamical matrix was constructed. Then, the vibrational modes were determined by a direct diagonalization of the dynamical matrix. The $k$-point set was increased step by step up to $24 \times 24 \times 1$ until the convergence for the frequencies of acoustical modes was reached $\left(0.0 \mathrm{~cm}^{-1}\right.$ for each acoustical mode). Once the accurate phonon mode frequencies were obtained, the change of macroscopic dielectric tensor was calculated with respect to each vibrational mode to get the corresponding Raman activities.

In order to investigate the response of the peak frequencies to the applied biaxial strain for Raman-active modes, the rate of change of the peak frequency and the corresponding mode Gruneissen parameter were calculated. The mode Gruneissen parameter at any wave vector $q$ can be calculated by the formula

$$
\gamma(q)=-\frac{a_{0}}{2 \omega_{0}(q)}\left[\frac{\omega_{+}(q)-\omega_{-}(q)}{a_{+}-a_{-}}\right],
$$

where $a_{0}$ is the relaxed (unstrained) lattice constant, $\omega_{0}(q)$ is the unstrained phonon frequency at wave vector $q, \omega_{+}(q)$ and $\omega_{-}(q)$ are the phonon frequencies under tensile and compressive biaxial strain, respectively, and $a_{+}-a_{-}$is the difference in the lattice constant when the system is under biaxial strain. In the present study, the phonon frequencies are calculated in the $q=0$ limit, at the $\Gamma$.

\section{STRAIN-DEPENDENT RAMAN ACTIVITY}

Basically in the Raman experiment, the sample is exposed to light and instantly scattered photons are collected. The dispersion of the collected photons with respect to shift in frequency gives the Raman spectrum. In the Raman theory, inelastically scattered photons originating from the oscillating dipoles of the crystal correspond to the Raman-active vibrational modes of the crystal.

The treatment of Raman intensities is based on Placzek's classical theory of polarizability. According to the classical
Placzek approximation, the activity of a Raman-active phonon mode is proportional to $\left|\hat{e}_{s} \cdot R \cdot \hat{e}_{i}\right|^{2}$ where $\hat{e}_{s}$ and $\hat{e}_{i}$ stand for the polarization vectors of scattered radiation and incident light, respectively. $R$ is a $3 \times 3$ second-rank tensor called a "Raman tensor" whose elements are the derivatives of polarizability of the material with respect to vibrational normal modes,

$$
R=\left[\begin{array}{lll}
\frac{\partial \alpha_{11}}{\partial Q_{k}} & \frac{\partial \alpha_{12}}{\partial Q_{k}} & \frac{\partial \alpha_{13}}{\partial Q_{k}} \\
\frac{\partial \alpha_{21}}{\partial Q_{k}} & \frac{\partial \alpha_{22}}{\partial Q_{k}} & \frac{\partial \alpha_{23}}{\partial Q_{k}} \\
\frac{\partial \alpha_{31}}{\partial Q_{k}} & \frac{\partial \alpha_{32}}{\partial Q_{k}} & \frac{\partial \alpha_{33}}{\partial Q_{k}}
\end{array}\right],
$$

where $Q_{k}$ is the normal mode describing the whole motion of individual atoms participating to the $k$ th vibrational mode and $\alpha_{i j}$ is the polarizability tensor of the material. The term $\mid \hat{e}_{s}$. $\left.R \cdot \hat{e}_{i}\right|^{2}$ is called the Raman activity which is calculated from the change of polarizability. For a backscattering experimental geometry, if orientational averaging is considered, the Raman activity is represented in terms of Raman invariants given by

$$
\begin{gathered}
\tilde{\alpha}_{s} \equiv \frac{1}{3}\left(\tilde{\alpha}_{x x}+\tilde{\alpha}_{y y}+\tilde{\alpha}_{z z}\right) \\
\beta \equiv \frac{1}{2}\left\{\left(\tilde{\alpha}_{x x}-\tilde{\alpha}_{y y}\right)^{2}+\left(\tilde{\alpha}_{y y}-\tilde{\alpha}_{z z}\right)^{2}+\left(\tilde{\alpha}_{z z}-\tilde{\alpha}_{x x}\right)^{2}\right. \\
\left.+6\left[\left(\tilde{\alpha}_{x y}\right)^{2}+\left(\tilde{\alpha}_{y z}\right)^{2}+\left(\tilde{\alpha}_{x z}\right)^{2}\right]\right\}
\end{gathered}
$$

where $\tilde{\alpha}_{s}$ and $\beta$ represent the isotropic and anisotropic parts of the derivative of polarizability tensor, respectively. The $\tilde{\alpha}$ represents the derivative of polarizability with respect to a normal mode. In the representation of the activity in terms of the variables, the activity is invariant under the orientation of the sample. Finally, using the forms of isotropic and anisotropic polarizability derivative tensors, the Raman activity $\mathrm{R}_{A}$ can be written as

$$
\mathrm{R}_{A}=45 \tilde{\alpha}^{2}+7 \beta^{2} \text {. }
$$

In fact, every Raman-active phonon mode has a finite scattering intensity which is measured in experiments. The term Raman activity $\mathrm{R}_{A}$ is directly related to the intensity through the following formula:

$$
I_{\text {Raman }}=\frac{I_{0} \pi^{2}}{45 \varepsilon_{0}^{2} \lambda^{4}} \mathrm{R}_{A},
$$

where $I_{0}$ is the intensity of incoming light, $\lambda$ is its wavelength, and $\mathrm{R}_{A}$ is the Raman activity. In the rest of the present study, Raman activities are discussed instead of intensities since the Raman activity is independent of the wavelength and the intensity of incoming light and is given in terms of Raman invariants [see Eq. (5)].

\section{A. Monoatomic single-layer crystals}

Monoatomic single layers of graphene, silicene, and germanene have hexagonal crystal structures. Due to $s p^{2}$ hybridization of $\mathrm{C}$ atoms in graphene, its structure is planar and belongs to $P \overline{6} / \mathrm{mmm}$ space-group symmetry. On the other hand, $s p^{3}$ hybridization in silicene and germanene results in a buckled geometry [see Fig. 1(a)]. The structure of the two buckled single layers belong to the $P \overline{3} m \overline{1}$ space group. Graphene, silicene, and germanene are known to exhibit a tiny electronic band gap of $24 \times 10^{-3}$ [51], 1.55-7.90 [52], and 24-93 meV [53,54], respectively (Table I). 


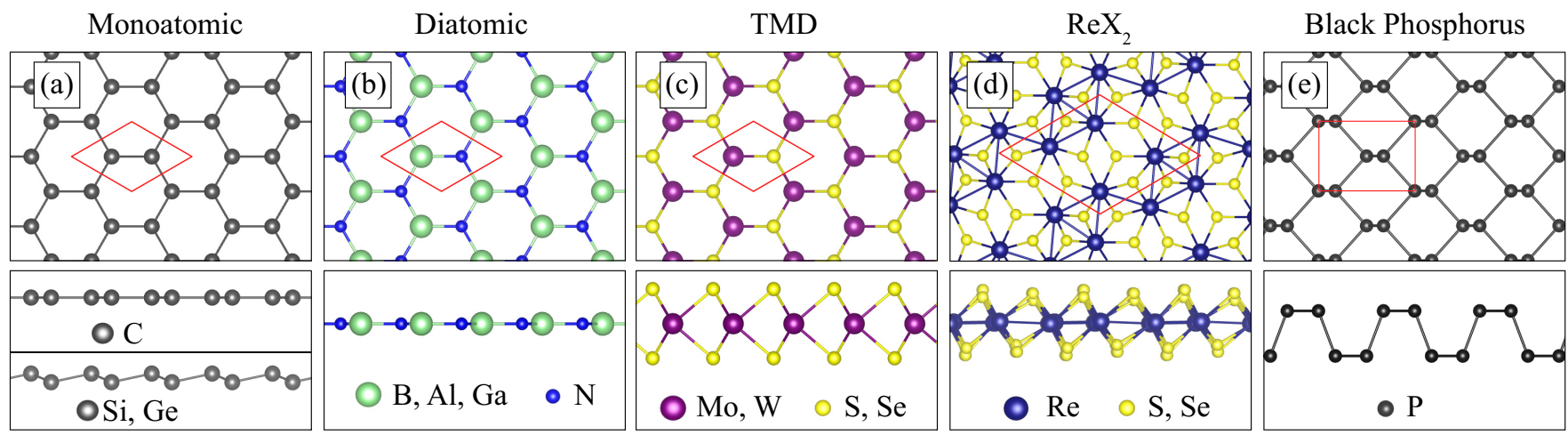

FIG. 1. Top and side view of single-layer crystal structures of (a) monoatomic, (b) diatomic, (c) TMDs, (d) Re $X_{2}$, and (e) bp. Color code of individual atoms are given in each figure. Primitive cells are indicated with red solid lines.

Graphene, silicene, and germanene exhibit six phonon branches that consist of three acoustical and three optical branches [see Fig. 2(a)]. One of the optical-phonon modes represents out-of-plane vibrational motion of the atoms and is named as an $A$-peak phonon mode. The other two optical modes have in-plane vibrational characteristics and are known as the $G$ peak in graphene and the $E$ peak in silicene and germanene. The $G$ and $E$ peaks are doubly degenerate at the $\Gamma$ due to in-plane isotropy of the crystals.

In the case of graphene, the frequency of the $A$ peak is $872.8 \mathrm{~cm}^{-1}$ and it is Raman inactive due to the planar crystal structure. On the other hand, the $G$ peak is a characteristic Raman-active mode in graphene and its frequency is $1555.0 \mathrm{~cm}^{-1}$. When an in-plane biaxial strain is applied to the crystal, a significant phonon softening (hardening) occurs under tensile (compressive) strain cases which is expected due to the positive mode Gruneissen parameter (see Table II). As shown in Fig. 3(a), the frequency of the $G$ peak softens to $1441.8 \mathrm{~cm}^{-1}$ at $2 \%$ of tensile strain while it hardens to $1675.1 \mathrm{~cm}^{-1}$ at $2 \%$ of compressive strain. The variation of
Raman activity of the $G$ peak is also shown in Fig. 3(a). It is seen that as the structure is biaxially stretched, the dipole between oppositely vibrating atoms gets larger, the polarizability increases, and hence the Raman activity increases. Contrarily, when the structure is compressed, the length of the dipole gets smaller and the Raman activity decreases.

The frequency of the $A$ peak is $193.8 \mathrm{~cm}^{-1}$ for silicene and due to the buckled structure the $A$ peak is found to be Raman active. The $E$-peak frequency is found at $559.6 \mathrm{~cm}^{-1}$ and is known to be another Raman-active mode in silicene. Experimentally, the $E$ peak is much more prominent than the $A$ peak due to its much higher Raman activity. The Raman activity of the $E$ peak is $10^{5}$ times of that of the $A$ peak. As shown in Fig. 3(b), when biaxial strain is applied, both peaks soften (harden) under tensile (compressive) strain cases. However, the response of Raman activities of $A$ and $E$ peaks is opposite. As the structure is stretched, the length of dipole in the $A$ peak gets smaller while it gets larger in the $E$ peak. Thus, the polarizability, hence the Raman activity, of the $A$ peak decreases while it increases for the $E$ peak. The situation is

TABLE I. For the single-layer crystal structures, the structure, planar (PL), low-buckled (LB), or puckered (P) calculated lattice parameters $a$ and $b$, the point group of the crystal, total number of Raman-active phonon modes, calculated in-plane static (low-frequency) dielectric constant $\epsilon_{\mathrm{cal}}$, previously reported in-plane static dielectric constant $\epsilon_{\mathrm{rep}}$, the energy-band gap of the structures calculated within SOC on top of $\operatorname{GGA}\left(E_{\mathrm{g}}^{\mathrm{SOC}}\right)$, for lateral orientations of the crystals; the in-plane stiffness, $C_{x}$ and $C_{y}$, and Poisson ratio, $v_{x}$ and $v_{y}$.

\begin{tabular}{|c|c|c|c|c|c|c|c|c|c|c|c|c|}
\hline & Structure & $\begin{array}{c}a \\
(\AA)\end{array}$ & $\begin{array}{c}b \\
(\AA)\end{array}$ & $\begin{array}{l}\text { Point } \\
\text { group }\end{array}$ & $\begin{array}{c}\text { No. of } \\
\text { Raman-active } \\
\text { modes }\end{array}$ & $\epsilon_{\mathrm{cal}}$ & $\epsilon_{\mathrm{rep}}$ & $\begin{array}{c}E_{g}^{\mathrm{SOC}} \\
(\mathrm{eV})\end{array}$ & $\begin{array}{c}C_{x} \\
(\mathrm{~N} / \mathrm{m})\end{array}$ & $\begin{array}{c}C_{y} \\
(\mathrm{~N} / \mathrm{m})\end{array}$ & $v_{x}$ & $v_{y}$ \\
\hline Graphene & PL & 2.47 & 2.47 & $D_{3 h}$ & 2 & 23.31 & & $\left(24 \times 10^{-6}\right)[51]$ & 330 & 330 & 0.19 & 0.19 \\
\hline Silicene & LB & 3.85 & 3.85 & $D_{3 d}$ & 3 & 8.01 & & $0.001-0.01[52]$ & 54 & 54 & 0.41 & 0.41 \\
\hline Germanene & LB & 4.01 & 4.01 & $D_{3 d}$ & 3 & 9.04 & & $0.02-0.1[53,54]$ & 38 & 38 & 0.42 & 0.42 \\
\hline$h-\mathrm{BN}$ & PL & 2.51 & 2.51 & $D_{3 h}$ & 2 & 1.5 & $2-4[55]$ & $4.68(d)$ & 273 & 273 & 0.22 & 0.22 \\
\hline$h-\mathrm{AlN}$ & PL & 3.13 & 3.13 & $D_{3 h}$ & 2 & 1.46 & & $3.61(i)$ & 112 & 112 & 0.46 & 0.46 \\
\hline$h-\mathrm{GaN}$ & PL & 3.27 & 3.27 & $D_{3 h}$ & 2 & 1.71 & & $2.37(i)$ & 109 & 109 & 0.48 & 0.48 \\
\hline $\mathrm{MoS}_{2}$ & $1 H$ & 3.19 & 3.19 & $D_{3 h}$ & 5 & 4.46 & $4.2-7.6[56]$ & $1.56(d)$ & 122 & 122 & 0.26 & 0.26 \\
\hline $\mathrm{MoSe}_{2}$ & $1 H$ & 3.32 & 3.32 & $D_{3 h}$ & 5 & 5.02 & $4.74[57]$ & $1.33(d)$ & 109 & 109 & 0.25 & 0.25 \\
\hline $\mathrm{WS}_{2}$ & $1 H$ & 3.18 & 3.18 & $D_{3 h}$ & 5 & 4.12 & 4.13 [57] & $1.53(d)$ & 122 & 122 & 0.21 & 0.21 \\
\hline $\mathrm{WSe}_{2}$ & $1 H$ & 3.33 & 3.33 & $D_{3 h}$ & 5 & 4.67 & 4.63 [57] & $1.19(d)$ & 99 & 99 & 0.20 & 0.20 \\
\hline $\mathrm{ReS}_{2}$ & $1 T^{\prime}$ & 6.46 & 6.38 & $C_{1 h}$ & 18 & $4.18^{a}$ & & $1.34(d)$ & 166 & 159 & 0.19 & 0.19 \\
\hline $\mathrm{ReSe}_{2}$ & $1 T^{\prime}$ & 6.71 & 6.60 & $C_{1 h}$ & 18 & $4.71^{\mathrm{a}}$ & & $1.27(d)$ & 138 & 138 & 0.18 & 0.18 \\
\hline bp & $\mathrm{P}$ & 4.57 & 3.31 & $D_{2 h}$ & 6 & $4.32^{\mathrm{a}}$ & & $0.88(d)$ & 91 & 29 & 0.65 & 0.21 \\
\hline
\end{tabular}

${ }^{a}$ The average in-plane static dielectric constant taken for anisotropic materials. 

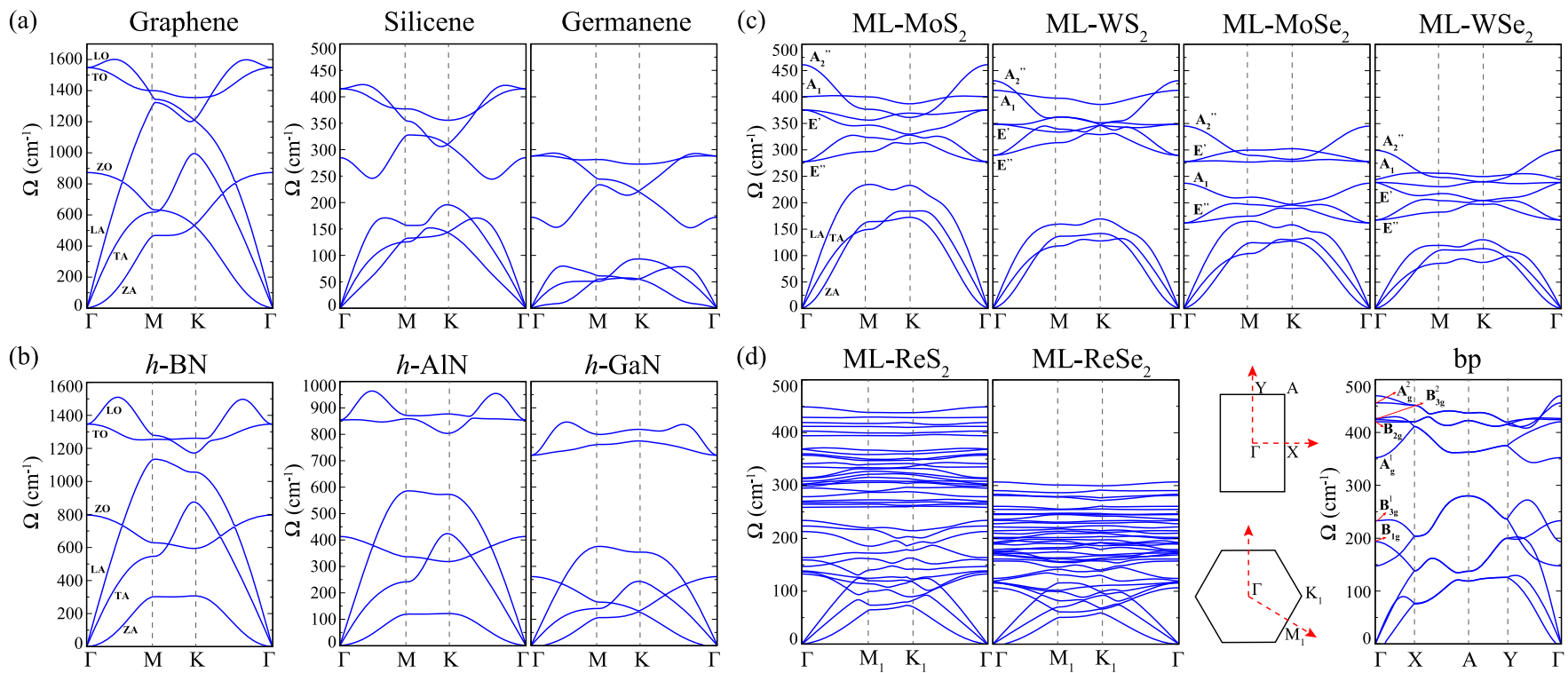

(d)

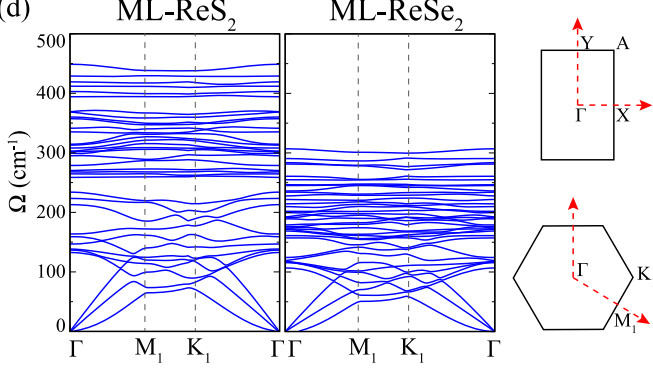

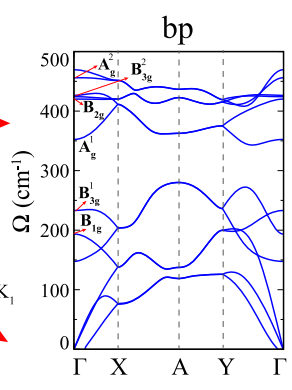

FIG. 2. Calculated phonon-band structures for single layers of (a) monoatomic, (b) diatomic, (c) isotropic TMDs, and (d) in-plane anisotropic crystals. Each vibrational phonon mode is named on the corresponding dispersion line. The Brillouin zone of in-plane anisotropic symmetry group is also shown.

reversed in the compressive strain case as shown in Fig. 3(b). The important point is that the Raman activity of the $A$ peak may disappear under high tensile strain values $(>2 \%)$ as the buckling of the structure decreases.

For the germanene, the peak position of the $A$ peak is at $172.2 \mathrm{~cm}^{-1}$. Since germanene is a softer material than silicene, the peak frequency of the $A$ peak is smaller. As in the case of silicene, the $A$ peak is Raman active for germanene due to the $s p^{3}$ hybridization of Ge atoms. The frequency of the $E$ peak is $296.8 \mathrm{~cm}^{-1}$ which is also much smaller than that in silicene. Both peaks display the same behavior for peak frequencies and corresponding Raman activities as in the case of silicene. As shown in Fig. 3(c), the $E$ peak displays a phonon softening to $279.4 \mathrm{~cm}^{-1}$ at $2 \%$ of tensile strain while it displays a phonon hardening to $312.2 \mathrm{~cm}^{-1}$ at $2 \%$ of compressive strain. In addition, the Raman activity of the $A$ peak decreases and disappears at $2 \%$ of tensile strain.

Each monoatomic single layer displays different responses to applied strain. The difference in the slope of the curves in Fig. 5(a) occurs due to the different mode Gruneissen

TABLE II. Unstrained peak frequency $\omega_{0}$, rate of change of peak frequency $\frac{1}{\omega} \frac{d \omega}{d \varepsilon}$, corresponding mode Gruneissen parameter $\gamma$, and reported value of mode Gruneissen parameter in previous studies, $\gamma_{\text {rep }}$, for mono- and diatomic single-layer crystals. Only the prominent and high-frequency peaks are considered.

\begin{tabular}{lrrrr}
\hline \hline & $\begin{array}{c}\omega_{0} \\
\left(\mathrm{~cm}^{-1}\right)\end{array}$ & $\begin{array}{c}\frac{1}{\omega} \frac{d \omega}{d \varepsilon} \\
(\%)\end{array}$ & $\gamma$ & $\gamma_{\text {rep }}$ \\
\hline Graphene & 1555.0 & 3.75 & 1.87 & $1.85[58]$ \\
Silicene & 559.6 & 2.10 & 1.05 & \\
Germanene & 296.8 & 2.78 & 1.39 & \\
$h$-BN & 1343.4 & 4.13 & 2.07 & $1.70[59]$ \\
$h$-AlN & 855.5 & 4.60 & 2.30 & \\
$h$-GaN & 721.6 & 4.84 & 2.42 & \\
\hline \hline
\end{tabular}

parameters $(\gamma)$ of the $G$ and $E$ peaks in each material. The $\gamma$ values are given in Table II and graphene has the largest $\gamma$ value which is a result of strong $\mathrm{C}-\mathrm{C}$ bonds in the crystal. As shown in Fig. 5(b), the amount of change of Raman activities is also different for each single-layer crystal. Stiff material, with strong interatomic bonds, charges uniformly distributed between the atoms are not localized in any region even at relatively high strains. Therefore, the change of dielectric constant hence the change of Raman activity is linear. However, in the case of silicene and germanene the charge is localized between the atoms and thus, as the strain is increased the Raman activity displays a nonlinear change [see Fig. 5(b)].

\section{B. Diatomic single-layer crystals}

Similar to the crystal structure of graphene, diatomic single layers of group-III nitrides ( $h$-BN, $h$-AIN, and $h$-GaN) have planar, one-atom-thick structure as shown in Fig. 1(b). The crystal structures belong to the space group $P 6_{3} / \mathrm{mmc}$. Single layers of the group-III nitrides exhibit six phonon branches [see Fig. 2(b)]. In addition to the $A$ peak, the doubly degenerate in-plane phonon mode $E_{2 g}$ is the optical phonon mode of the single layers. The $A$ peak is Raman-inactive mode for the group-III nitrides while $E_{2 g}$ is the prominent peak.

The frequency of the $A$ peak is $800.9 \mathrm{~cm}^{-1}$ for $h$-BN while the frequency of $E_{2 g}$ is found at $1343.4 \mathrm{~cm}^{-1}$. Due to the occupied in-plane orbitals in the crystal, the frequency of $E_{2 g}$ is much greater than that of the $A$ peak. Under biaxial strain, the $E_{2 g}$ peak reveals a softening to $1235.5 \mathrm{~cm}^{-1}$ at $2 \%$ stretching and displays a hardening to $1457.7 \mathrm{~cm}^{-1}$ at $2 \%$ compression. The Raman activity of the $E_{2 g}$ peak increases with increasing tensile strain while it decreases with increasing compressive strain as shown in Fig. 4(a). As in the case of graphene, the change of Raman activity is also linear for $h$-BN due to the strong B-N bonds which preserves the charge distribution even at high strains. The response of Raman-inactive $A$ peak to the applied strain is not discussed. 

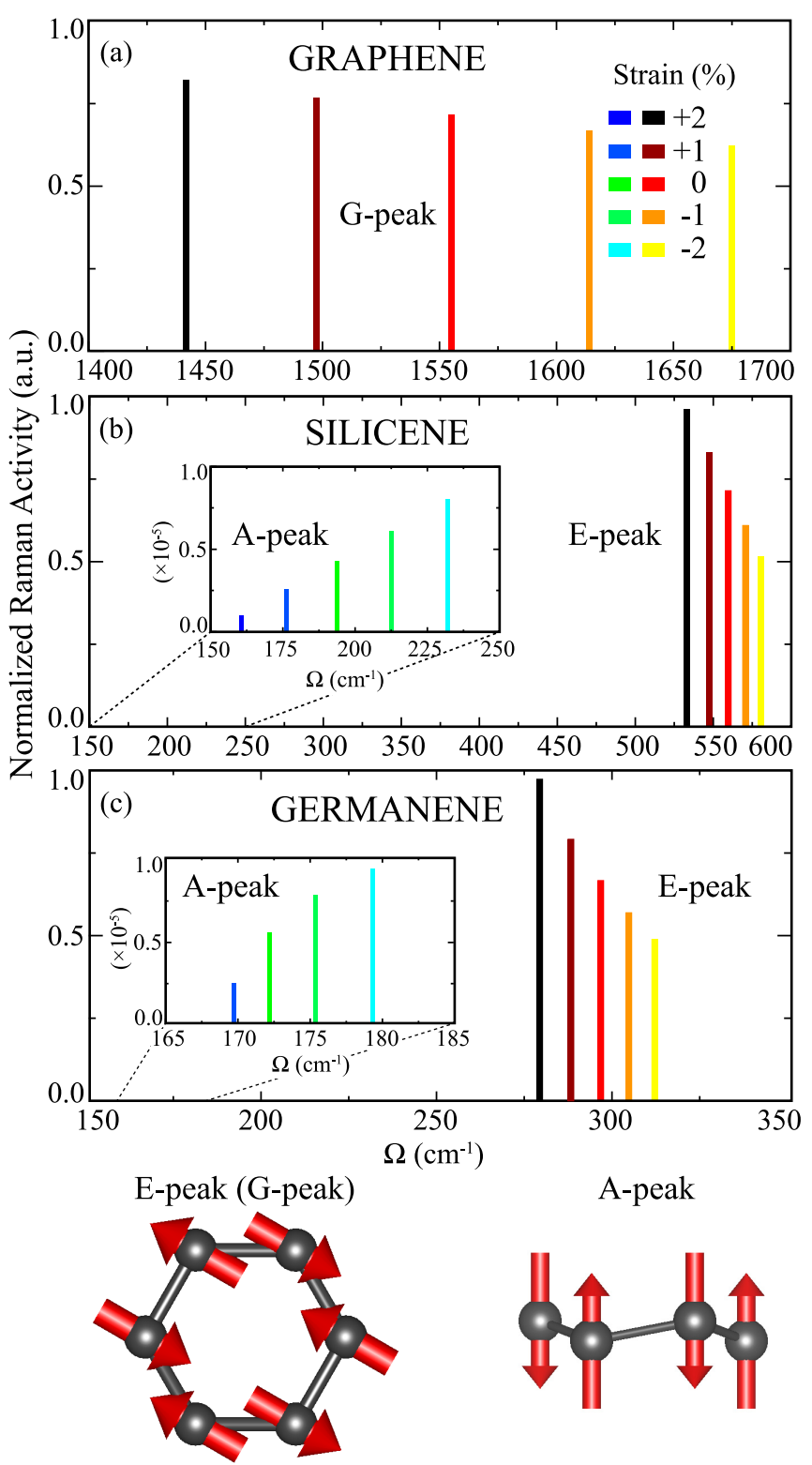

FIG. 3. The response of activity of Raman-active modes to the applied biaxial strain for (a) graphene, (b) silicene, and (c) germanene. The insets in (b) and (c) are given for $A$-peak phonon mode.

In the case of $h-\mathrm{AlN}$, the frequency of the $A$ peak softens to $412.5 \mathrm{~cm}^{-1}$ since the stiffness is smaller than that of $h$-BN. The frequency of $E_{2 g}$ is $855.5 \mathrm{~cm}^{-1}$ which is also smaller than that of the $h$-BN due to the same reason. When the single-layer $h$-AlN is biaxially stretched, the peak position of $E_{2 g}$ softens to $779.4 \mathrm{~cm}^{-1}$ while it hardens to $936.9 \mathrm{~cm}^{-1}$. In addition, the Raman activity displays the same trend as in the case of $h-\mathrm{BN}$ but the amount of change is different as shown in Fig. 5(b). The atomic bond length is much larger in $h$-AlN than in $h$-BN, thus increasing strain causes nonlinear change in the Raman activity of $h$-AlN because of the localized charge densities in the crystal.

The lowest frequencies of both the $A$ peak and the $E_{2 g}$ mode are found for single-layer $h-\mathrm{GaN}$ since it is the most flexible material through all considered group-III nitrides. The peak

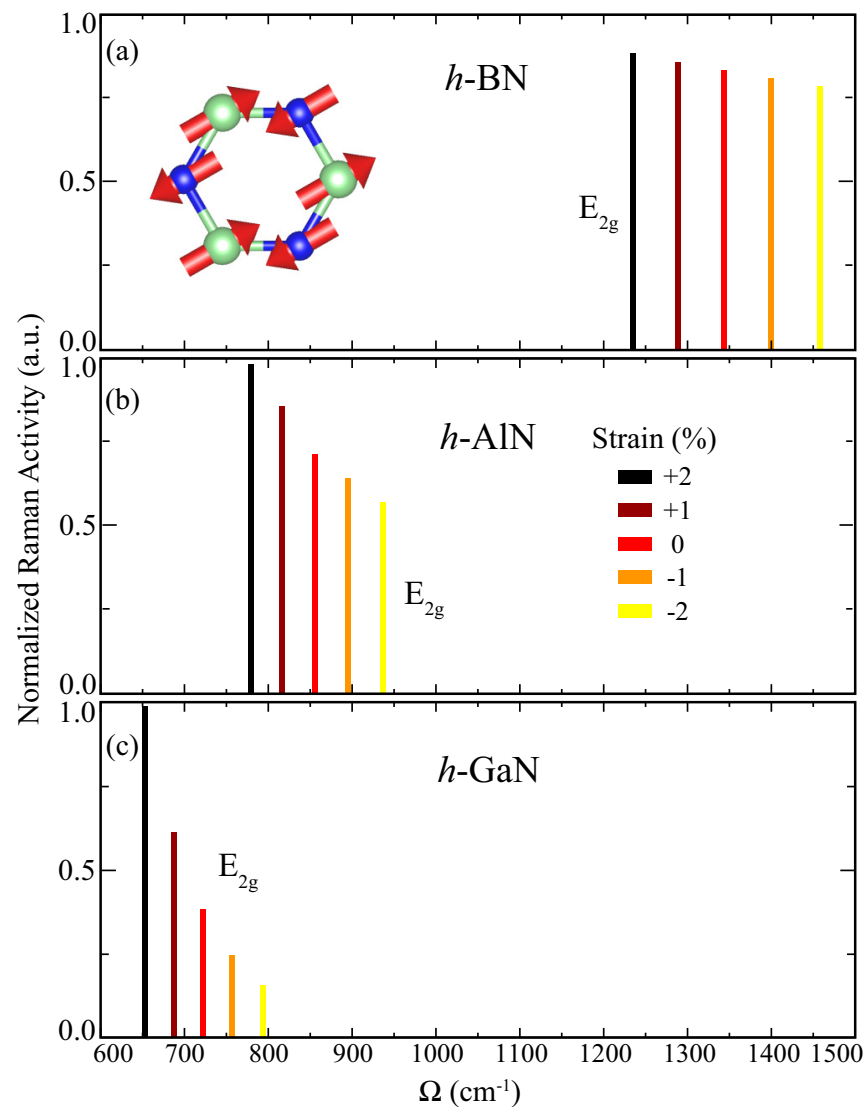

FIG. 4. The effect of biaxial strain on both the peak frequency and the corresponding Raman activity of the $E_{2 g}$ phonon modes for single layer; (a) $h$-BN, (b) $h$-AlN, and (c) $h-\mathrm{GaN}$. The vibrational motion of individual atoms in $E_{2 g}$ phonon mode is shown in the inset.

positions of the two modes are 263.6 and $721.6 \mathrm{~cm}^{-1}$ for the $A$ peak and $E_{2 g}$, respectively. The highest rate of change of peak frequency with respect to the unstrained frequency is found for $h$-GaN as given in Table II by the mode Gruneissen parameter.

As shown in Fig. 5(b), the response of Raman activity of each material differs as the applied strain increases. As mentioned in the theory part, the Raman activity is a function of $\tilde{\alpha}^{2}$ which depends on static dielectric constant $\epsilon$ and the $\epsilon$ is a function of square of charges on the atoms. Graphene and $h$-BN display linear response even at $\pm 2 \%$ strain [see Fig. 5(b)]
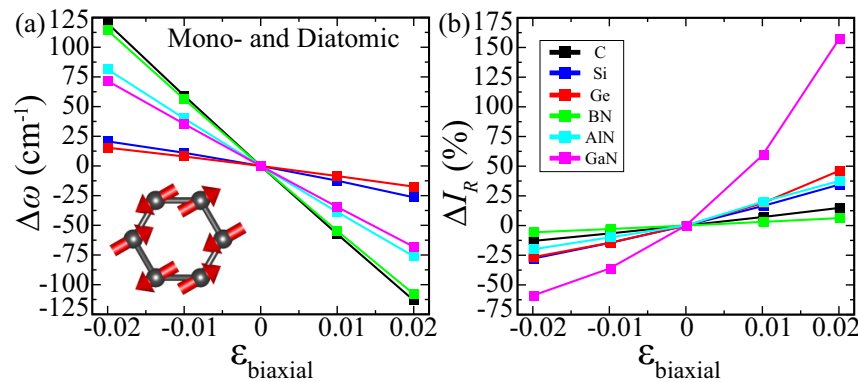

FIG. 5. (a) The response of peak positions and (b) corresponding Raman activities to the applied biaxial strain for single layers of monoand diatomic crystal structures for $G$ peak, $E$ peak, and $E_{2 g}$ mode. 
due to the strong $\mathrm{C}-\mathrm{C}$ and $\mathrm{B}-\mathrm{N}$ bonds which preserve charge distribution in the crystal. As the in-plane stiffness decreases, the conservation of charge distribution is not possible with increasing strain value. Thus, silicene and germanene exhibit nonlinear response out of the strain range $\pm 1 \%$. Moreover, special to the case of $h$-GaN, the charges are initially localized on $\mathrm{N}$ atoms which indicates relatively weak $\mathrm{Ga}-\mathrm{N}$ bonding in the crystal. Therefore, even at small strains, the Raman activity of $h$-GaN displays nonlinear responses.

\section{TMDs single-layer crystals}

The single layers of TMDs have a hexagonally packed crystal structure in which the sublayer of metal $(M)$ atom is sandwiched between two sublayers of chalcogen $(X)$ atoms [see Fig. 1(d)]. Most of the single-layer TMDs crystallize in either $1 H$ or $1 T$ phases. Here, we consider single layers of Mo and W dichalcogenides which have $1 H$ crystal structure. Single-layer crystals of the TMDs exhibit $P \overline{6} / m \overline{2}$ space-group symmetry. $M X_{2}(M=\mathrm{Mo}$ or $\mathrm{W}, X=\mathrm{S}$ or $\mathrm{Se})$ crystals exhibit nine phonon branches, six of which are optical phonon branches [see Fig. 2(c)]. The group theory analysis indicates that due to the $D_{3 h}$ symmetry group there are five Raman-active modes. The in-plane optical phonon modes are known as the $E^{\prime}$ and $E^{\prime \prime}$ which are both doubly degenerate and Raman active. Additionally, an out-of-plane optical mode, $A_{1}$, is Raman active (see Fig. 6). Another out-of-plane optical mode, $A_{2}^{\prime \prime}$, is the only Raman inactive mode for the single-layer TMDs.

The $E^{\prime \prime}$ phonon mode is the lowest frequency optical mode which reveals only the vibration of the chalcogen atoms in opposite directions. The frequencies of $E^{\prime \prime}$ are 277.8, 162.5, 289.5, and $167.9 \mathrm{~cm}^{-1}$ for $\mathrm{MoS}_{2}, \mathrm{MoSe}_{2}, \mathrm{WS}_{2}$, and $\mathrm{WSe}_{2}$, respectively. Since the mass of the Se atom is much larger than that of the $\mathrm{S}$ atom, phonon modes soften in $\mathrm{MSe}_{2}$ crystals. The Raman activity of the $E^{\prime \prime}$ mode is much smaller than those of the $E^{\prime}$ and $A_{1}$ modes in each single layer. In $M S_{2}$ crystals its Raman activity is in the order of $10^{-4}$ while in $M \mathrm{Se}_{2}$ structures the activity is in the order of $10^{-3}$. Thus, it is not a prominent Raman peak in the experiments.

When the crystals are biaxially stretched, $E^{\prime \prime}$ displays phonon softening and demonstrates a phonon hardening under compressive strains as shown in Fig. 6. The corresponding mode Gruneissen parameters are given in Table III. Since the Raman activity of $E^{\prime \prime}$ is very small, it is still not prominent even at high tensile strains. Because of the nonsignificant changes in the Raman activity of $E^{\prime \prime}$, two other prominent Raman peaks are discussed which may determine the strain in single-layer TMDs.

The $E^{\prime}$ mode demonstrates the vibration of the transitionmetal atom in the opposite direction to the chalcogen atoms. The frequencies of $E^{\prime}$ are $375.8,278.3,348.2$, and $239.0 \mathrm{~cm}^{-1}$ for $\mathrm{MoS}_{2}, \mathrm{MoSe}_{2}, \mathrm{WS}_{2}$, and $\mathrm{WSe}_{2}$, respectively. The Raman activity of $E^{\prime}$ indicates that it is a prominent peak clearly observed in experiments [60]. The response of the frequency of the $E^{\prime}$ peak to the biaxial strain is discussed through the calculated mode Gruneissen parameter for each single layer. As given in Table III, $\gamma$ values are 0.68 (0.65) [61], 0.53, 0.64, and 0.54 for $\mathrm{MoS}_{2}, \mathrm{MoSe}_{2}, \mathrm{WS}_{2}$, and $\mathrm{WSe}_{2}$, respectively. The Raman activity of the $E^{\prime}$ peak demonstrates a similar response to the biaxial strain with that in monoatomic and diatomic
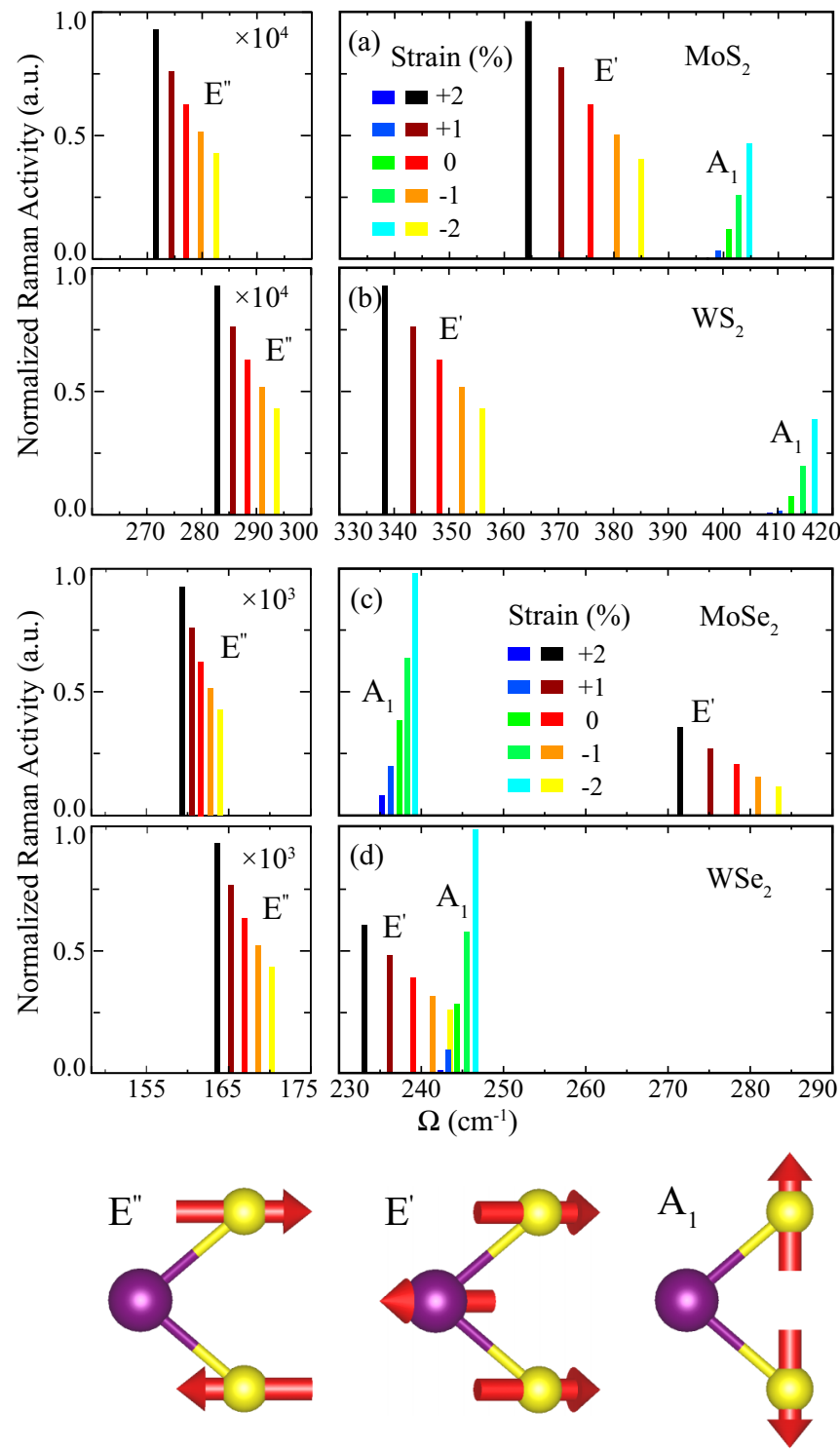

FIG. 6. The response of Raman-active modes to the applied biaxial strain for single-layer (a) $\mathrm{MoS}_{2}$, (b) $\mathrm{WS}_{2}$, (c) $\mathrm{MoSe}_{2}$, and (d) $\mathrm{WSe}_{2}$. The vibrational motions of atoms in corresponding phonon modes are shown below.

cases. Due to opposite responses of $E^{\prime}$ and $A_{1}$ peaks, discussion of their relative Raman activities is more meaningful.

As mentioned above, the $A_{1}$ phonon mode is the only Raman-active out-of-plane optical mode. In all the single-layer TMDs the frequency of the $A_{1}$ mode is higher than that of $E^{\prime}$ except for $\mathrm{MoSe}_{2}$. The frequencies of $A_{1}$ are 401.0, 237.3, 412.4, and $244.4 \mathrm{~cm}^{-1}$ for single-layer $\mathrm{MoS}_{2}, \mathrm{MoSe}_{2}$, $\mathrm{WS}_{2}$, and $\mathrm{WSe}_{2}$, respectively. The higher frequencies in $M \mathrm{~S}_{2}$ crystals are due to the smaller vertical distance of S-S atoms. In the unstrained single-layer TMDs, the Raman activity of the $A_{1}$ peak is smaller than that of $E^{\prime}$ except for $\mathrm{MoSe}_{2}$ (see Fig. 6). In $\mathrm{vdW}$ layered materials, increasing number of layers strongly affects the activity of the $A_{1}$ peak due to additional interlayer interaction. As given in Table III, $\gamma$ values for the $A_{1}$ peak are smaller than the values for $E^{\prime}$ which means the frequency of $A_{1}$ is less affected by the in-plane strain. This is meaningful 
TABLE III. Unstrained peak frequency $\omega_{0}$, rate of change of peak frequency $\frac{1}{\omega} \frac{d \omega}{d \varepsilon}$, corresponding mode Gruneissen parameter $\gamma$, for in-plane isotropic single-layer TMDs.

\begin{tabular}{|c|c|c|c|c|c|c|c|c|c|c|c|}
\hline$E^{\prime \prime}$ & $\begin{array}{c}\omega_{0} \\
\left(\mathrm{~cm}^{-1}\right)\end{array}$ & $\begin{array}{l}\frac{1}{\omega} \frac{d \omega}{d \varepsilon} \\
(\%)\end{array}$ & $\gamma$ & $E^{\prime}$ & $\begin{array}{c}\omega_{0} \\
\left(\mathrm{~cm}^{-1}\right)\end{array}$ & $\begin{array}{c}\frac{1}{\omega} \frac{d \omega}{d \varepsilon} \\
(\%)\end{array}$ & $\gamma$ & $A_{1}$ & $\begin{array}{c}\omega_{0} \\
\left(\mathrm{~cm}^{-1}\right)\end{array}$ & $\begin{array}{c}\frac{1}{\omega} \frac{d \omega}{d \varepsilon} \\
(\%)\end{array}$ & $\gamma$ \\
\hline $\mathrm{MoS}_{2}$ & 277.8 & 1.04 & 0.52 & & 375.8 & 1.36 & 0.68 & & 401.0 & 0.46 & 0.23 \\
\hline $\mathrm{MoSe}_{2}$ & 162.2 & 0.77 & 0.39 & & 278.3 & 1.06 & 0.53 & & 237.3 & 0.42 & 0.21 \\
\hline $\mathrm{WS}_{2}$ & 289.5 & 0.98 & 0.49 & & 348.2 & 1.27 & 0.64 & & 412.4 & 0.51 & 0.25 \\
\hline $\mathrm{WSe}_{2}$ & 167.9 & 0.77 & 0.39 & & 239.0 & 1.08 & 0.54 & & 244.4 & 0.44 & 0.22 \\
\hline
\end{tabular}

since $A_{1}$ represents the out-of-plane vibration of the atoms. The values for $A_{1}$ are $0.23(0.21$ [61]), 0.21, 0.25, and 0.22 , respectively. In contrast to the response of Raman activity of $E^{\prime}$ to applied strain, the activity of $A_{1}$ increases when the structure is compressed which is a result of increasing dipole length between vibrating chalcogen atoms when structure is compressed in the in-plane directions (Fig. 7).

Although the Raman activity of both prominent peaks changes under biaxial strain, it is meaningful to discuss their relative ratios to identify the strain in the crystal. The ratios $\frac{I_{E^{\prime}}}{I_{A_{1}}}$ in the unstrained structures are $5.35,0.53,8.32$, and 1.37 for $\mathrm{MoS}_{2}, \mathrm{MoSe}_{2}, \mathrm{WS}_{2}$, and $\mathrm{WSe}_{2}$, respectively. Since $A_{1}$ and $E^{\prime}$ demonstrate opposite responses to the biaxial strain, the ratio gets much higher when the structure in stretched. This is an important point for the identification of biaxial strain on the crystal. For the maximum compression $(-2 \%)$ the ratios are $0.87,0.12,1.11$, and 0.26 , respectively. Contrary to compressive strain, the values are enhanced under the tensile strain case which is a strong indication of the stretched crystal. In the case of $M S_{2}$ crystals, $\frac{I_{E^{\prime}}}{I_{A_{1}}}$ increases to 574 and 204 for $\mathrm{MoS}_{2}$ and $\mathrm{WS}_{2}$, respectively. High values of the activity ratio can be clearly observed in a Raman experiment in which the samples are under tensile biaxial strain.
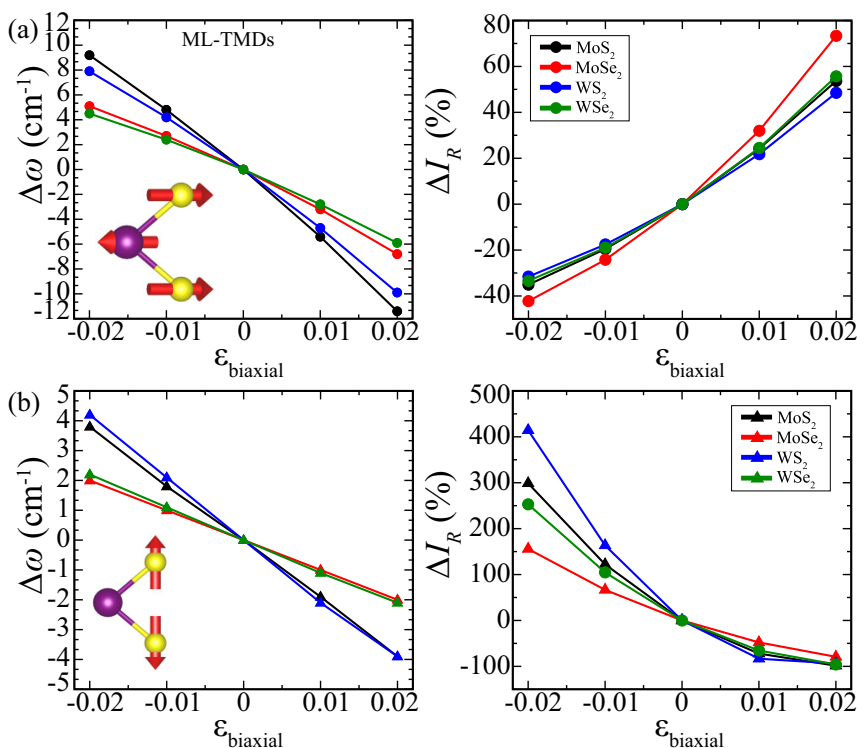

FIG. 7. The response of peak positions of Raman-active modes and their Raman activities to the applied biaxial strain for single layers of TMDs for (a) $E^{\prime}$ mode and (b) $A_{1}$.

\section{Anisotropic single-layer crystals}

\section{Rhenium dichalcogenides $\left(\operatorname{ReS}_{2}\right.$ and $\left.\operatorname{ReSe}_{2}\right)$ single-layer crystals}

Besides the perfect hexagonal lattice, there are also in-plane anisotropic single-layer crystals such as $\mathrm{ReS}_{2}, \mathrm{ReSe}_{2}$, and bp. As shown in Fig. 1(e), single layers of $\mathrm{ReS}_{2}$ and $\mathrm{ReSe}_{2}$ have distorted $1 T\left(1 T^{\prime}\right)$ crystal structure, which belongs to the space group $P \overline{1}$ [10]. A unit cell of the $1 T^{\prime}$ phase consists of eight chalcogen atoms coordinated around a diamondlike $\mathrm{Re}_{4}$ cluster which is formed by the strong interaction between $\mathrm{Re}$ atoms. The angle between the in-plane unit-cell vectors is $61.1^{\circ}$ due to distortion in the crystal structure.

For single-layer $\operatorname{Re} X_{2}$ there are 36 phonon modes as shown in Fig. 2(d). Due to the distorted and anisotropic crystal structure, all of the Raman-active phonon modes are nondegenerate. The 18 of the 36 phonon modes for both crystals are known to be Raman active from group theory [62].

The 18 Raman-active phonon modes of single-layer $\mathrm{ReS}_{2}$ are classified as $A_{g}$ like (representing out-of-plane motion of atoms), $E_{g}$ like (representing in-plane motion of atoms), and the coupled vibrations of the atoms in both directions. There are four $A_{g}$-like modes, two of which contain the motion of $\mathrm{Re}$ atoms while the other two contain the motion of $\mathrm{S}$ atoms. The modes with frequencies 132.6 and $139.9 \mathrm{~cm}^{-1}$ represent the $A_{g}$-like modes of Re atoms while the modes at 429.0 and $402.7 \mathrm{~cm}^{-1}$ represent those of $\mathrm{S}$ atoms. The Raman activities of $A_{g}$-like modes of $\mathrm{Re}$ are smaller than those for the modes of $\mathrm{S}$ atoms which is because of the smaller dipole length between Re atoms. The frequencies and the Raman activities of $A_{g}$-like modes of both atoms demonstrate similar behavior under applied strain as in other TMDs discussed in Sec. IIIC. However, the change of activity of $A_{g}$-like modes of $\mathrm{Re}$ is much smaller than that of $\mathrm{S}$ atoms because at the strength of applied strain the length of Re-Re dipoles is still small due to their strong interaction.

The total number of $E_{g}$-like modes is 6; four of the modes reveal the in-plane motion of Re atoms while the other two demonstrate that of $\mathrm{S}$ atoms. The frequencies indicate that the motion of $\mathrm{Re}$ atoms occurs at lower frequencies than those of $\mathrm{S}$ atoms. The frequencies of $E_{g}$-like modes of $\mathrm{Re}$ atoms are 151.3, 165.0, 218.9, and $239.5 \mathrm{~cm}^{-1}$, and 298.2 and $307.0 \mathrm{~cm}^{-1}$ for those of $\mathrm{S}$ atoms. The response of the in-plane modes to the applied strain for both peak positions and Raman activities agree with those for isotropic TMDs (see the dashed green lines in Fig. 8).

The remaining eight Raman-active modes represent the coupled vibration through in-plane and out-of-plane directions. 


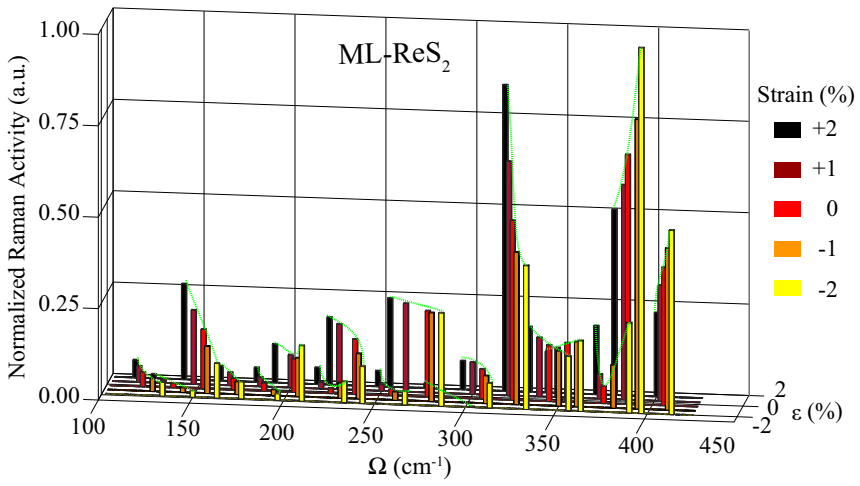

FIG. 8. The evolution of peak frequency of 18 Raman-active phonon modes and their corresponding Raman activities under biaxial strain in single-layer $\mathrm{ReS}_{2}$. Dashed green lines display the shift of the peak frequencies.

Six of the modes represent the vibration of only S atoms while in the other two modes coupled vibrations of Re and $\mathrm{S}$ atoms occur. Since for the responses of Raman activities of $A_{g}$-like and $E_{g}$-like modes an opposite trend is seen, the changes of the activity of coupled modes is found to be smaller. The change of Raman activity is determined by the prepotency of vibrations of atoms; that is, for example, if the in-plane motion is dominant to out of plane then we see an increase in the activity under the stretching of the crystal. The most significant change occurs for the coupled mode with frequency of $412.0 \mathrm{~cm}^{-1}$. In the mode, the out-of-plane vibrational motion is dominant to that of in-plane and thus the activity decreases under tensile strain at a rate of $36 \%$ while it increases at a rate of $47 \%$ under compression.

In the case of single-layer $\mathrm{ReSe}_{2}$, the frequencies of the Raman-active modes significantly soften when compared with those of $\mathrm{ReS}_{2}$ due to lower in-plane stiffness of the crystal. The highest Raman-active mode has a frequency of $290.7 \mathrm{~cm}^{-1}$. The $A_{g}$-like phonon modes of Re atoms have the frequencies of 106.5 and $116.0 \mathrm{~cm}^{-1}$ while the frequencies are 157.0 and $176.2 \mathrm{~cm}^{-1}$ for those of Se atoms. The most significant response of the Raman activity is found for the most intense peak at $260.9 \mathrm{~cm}^{-1}$ (see Fig. 9). Under compressive strain,

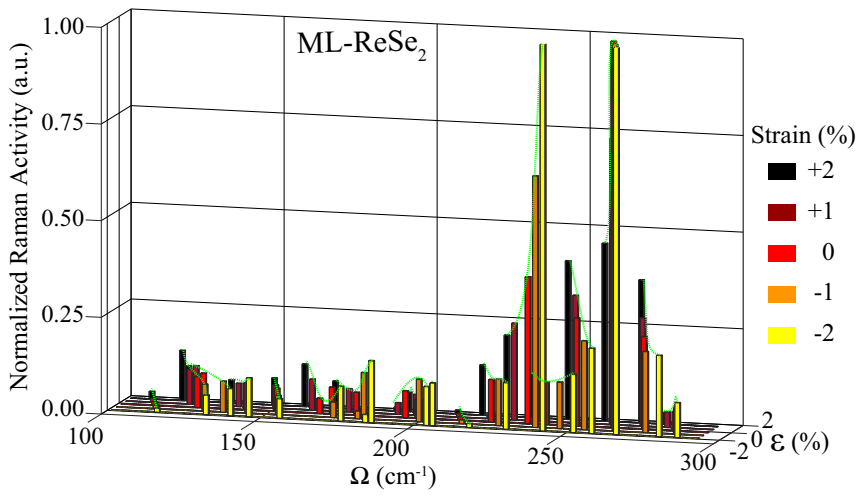

FIG. 9. The evolution of peak frequency of 18 Raman-active phonon modes and their corresponding Raman activities under biaxial strain in single-layer $\mathrm{ReSe}_{2}$. Dashed green lines display the shift of the peak frequencies. the activity of the coupled mode of Re and Se atoms increases about $50 \%$ of its unstrained value while it decreases about $55 \%$ under tensile biaxial strain. Although some of the Ramanactive modes display significant changes under strain, due to the rigidity of the $\operatorname{Re} X_{2}$ crystals they are almost irresponsive to the applied strain.

\section{Black phosphorus single-layer crystal}

Single-layer bp is a recently synthesized, in-plane anisotropic member of the 2D single-layer family. It is known to possess remarkable in-plane anisotropic electrical, optical, and phonon properties $[63,64]$. There are four $\mathrm{P}$ atoms in its rectangular primitive unit cell and its crystal structure belongs to the $C m c a$ space group.

The phonon-band structure of single-layer bp demonstrates that 12 phonon branches exist [see Fig. 2(d)]. According to group theory analysis, it exhibits six Raman-active phonon modes known as $B_{1 g}, B_{3 g}^{1}, A_{g}^{1}, B_{3 g}^{2}, B_{2 g}$, and $A_{g}^{2}$. The Raman-active modes, $B_{1 g}, B_{3 g}^{1}$, and $B_{2 g}$, represent the in-plane vibration of $\mathrm{P}$ atoms while $B_{3 g}^{2}, A_{g}^{1}$, and $A_{g}^{2}$ represent out-ofplane motion. Experimental measurements revealed that only three of the six Raman-active modes $\left(A_{g}^{1}, B_{2 g}\right.$, and $\left.A_{g}^{2}\right)$ exhibit prominent Raman intensity $[65,66]$. The frequencies of the three prominent peaks are $352.1,424.2$, and $455.2 \mathrm{~cm}^{-1}$ for $A_{g}^{1}, B_{2 g}$, and $A_{g}^{2}$, respectively. For the unstrained single-layer bp, Raman activities demonstrate that the $A_{g}^{1}$ and $A_{g}^{2}$ have much higher activities than that of $B_{2 g}$ which was also observed in the experiment $[65,66]$.

When single-layer bp is biaxially compressed up to $2 \%$, the frequency of the $A_{g}^{2}$ phonon mode displays a hardening from 455.2 to $462.6 \mathrm{~cm}^{-1}$ while a softening to $446.9 \mathrm{~cm}^{-1}$ is found under $2 \%$ tensile biaxial strain. The frequency of the $B_{2 g}$ phonon mode displays the same trend as the $A_{g}^{2}$ mode under biaxial strain. A softening to $403.5 \mathrm{~cm}^{-1}$ and a hardening to $442.9 \mathrm{~cm}^{-1}$ are seen for the frequency of the $B_{2 g}$ phonon mode under tensile and compressive strains, respectively. For the strain range of $\pm 2 \%$, the response of frequency of $B_{2 g}$ is much larger than that of the $A_{g}^{2}$ mode. Another characteristic Raman-active mode of single-layer bp is $A_{g}^{1}$ which represents the out-of-plane vibrations of the $\mathrm{P}$ atoms in upper and lower sublayers in opposite directions. The frequency of the mode softens to $343.7 \mathrm{~cm}^{-1}$ under $2 \%$ of tensile strain while it hardens to $358.2 \mathrm{~cm}^{-1}$ under that of compressive strain. The responses of peak frequencies of the three prominent modes to the applied in-plane biaxial strain are compared through their mode Gruneissen parameters given in Table IV. The greater $\gamma$ is calculated for $B_{2 g}$ mode which exactly has the in-plane vibrational characteristic. The $\gamma$ values of $A_{g}^{1}$ and $A_{g}^{2}$

TABLE IV. Unstrained peak frequency $\omega_{0}$, the rate of change of peak frequency $\frac{1}{\omega} \frac{d \omega}{d \varepsilon}$, and corresponding mode Gruneissen parameter $\gamma$ for three high-frequency prominent peaks in single-layer bp.

\begin{tabular}{lccc}
\hline \hline & $\omega_{0}\left(\mathrm{~cm}^{-1}\right)$ & $\frac{1}{\omega} \frac{d \omega}{d \varepsilon}(\%)$ & $\gamma$ \\
\hline$A_{g}^{1}$ & 352.1 & 1.05 & 0.53 \\
$B_{2 g}$ & 559.6 & 2.32 & 1.16 \\
$A_{g}^{2}$ & 296.8 & 0.87 & 0.44 \\
\hline \hline
\end{tabular}



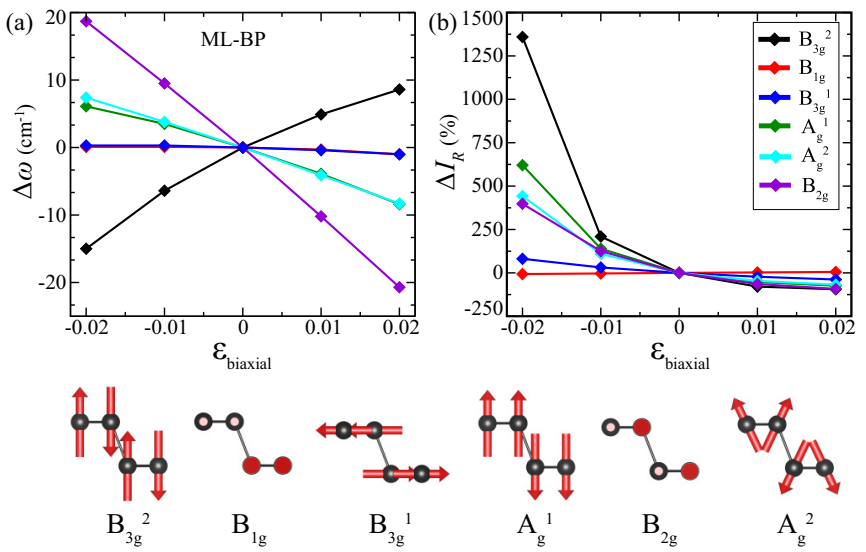

FIG. 10. (a) The response of frequencies and (b) Raman activities of six Raman-active modes of single-layer bp to the biaxial strain. Vibrational motion of $\mathrm{P}$ atoms in each phonon mode are given below.

are smaller than that of $B_{2 g}$ since they both demonstrate the out-of-plane vibration of $\mathrm{P}$ atoms (see Fig. 10).

As shown in Fig. 11, the Raman activity of the $A_{g}^{2}$ phonon mode is highly affected by the applied strain. The Raman activity of $A_{g}^{2}$ increases up to $5.4(440 \%)$ of its unstrained value under $2 \%$ of compressive strain. However, its Raman activity is less sensitive to tensile strain when compared with that of compressive strain. Under $2 \%$ of tensile strain, the Raman activity decreases about $68 \%$ of its initial value which is much lower than that of compressive value. In addition, the Raman activity of $B_{2 g}$ phonon mode is approximately 375 times smaller than that of the $A_{g}^{2}$ mode for the unstrained crystal structure. The Raman activity of $B_{2 g}$ displays a symmetric response under compressive and tensile biaxial strains. Due to the out-of-plane nature of the mode, the same trend is also illustrated for the $B_{2 g}$ mode. The Raman activity of the $A_{g}^{1}$ phonon mode is in the order of that of the $A_{g}^{2}$ mode in an unstrained structure. Since the two modes display the same trend under biaxial strain, it is meaningful to compare the Raman activity of $A_{g}^{1}$ with its unstrained value. When $2 \%$ of compressive strain is applied, the Raman activity of $A_{g}^{1}$ increases about seven times of its unstrained value.

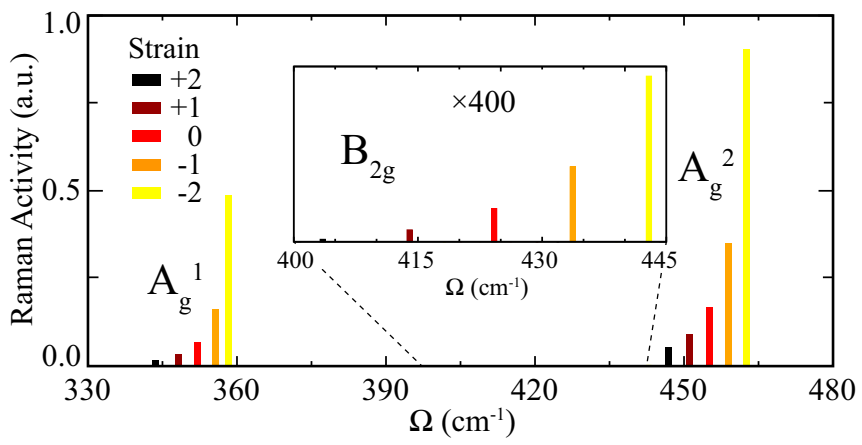

FIG. 11. The evolution of peak frequency of three prominent Raman-active phonon modes and their corresponding Raman activities under biaxial strain in single-layer bp.
As mentioned above, six total Raman-active modes exist for single-layer bp, three of which have very low Raman activities (at the order of $10^{-5}$ of the value of the $A_{g}^{2}$ mode). The frequencies of $B_{1 g}$ and $B_{3 g}^{1}$ are 193.8 and $232.8 \mathrm{~cm}^{-1}$, respectively. We find that both the frequencies and the corresponding Raman activities are mostly insensitive to the applied biaxial strain. The reason is the vibrational characteristic of the modes. It is seen that the modes represent in-plane vibration of $\mathrm{P}$ atoms. Differing from $B_{2 g}$, in $B_{1 g}$ and $B_{3 g}^{1} \mathrm{P}$ atoms located at the same layer vibrate in the same direction. As the biaxial strain is applied, the thickness of the layer decreases but the out-of-plane symmetry of the vibrating atoms is mostly conserved. Thus, both peak positions and Raman activities mostly remain unaffected by the applied strain.

\section{CONCLUSIONS}

In this study, the first-order off-resonant Raman spectra of 2D single-layers of monoatomic (graphene, $\mathrm{Si}$, and $\mathrm{Ge}$ ), diatomic ( $h$-BN, $h$-AlN, and $h$-GaN), in-plane isotropic TMDs $\left(\mathrm{MoS}_{2}, \mathrm{MoSe}_{2}, \mathrm{WS}_{2}\right.$, and $\left.\mathrm{WSe}_{2}\right)$, and in-plane anisotropic crystals $\left(\mathrm{ReS}_{2}, \mathrm{ReSe}_{2}\right.$, and bp) and their strain-dependent behaviors were investigated by performing DFT-based calculations. Our results fit well into the reported experimental results for the first-order off-resonant Raman activities. In addition, the effect of biaxial strain on Raman spectra of the single-layer crystals was analyzed in terms of their peak frequencies and corresponding Raman activities. Our findings can be summarized as follows: (i) strain can be directly observed in Raman-scattering experiments by the knowledge of the peak positions of Raman-active phonon modes, (ii) the $A$ peak of the single-layer Si and Ge disappear under sufficient tensile strain, (iii) especially in mono- and diatomic single layers, the shift of the peak frequencies is a stronger indication of the strain rather than the change in Raman activities, (iv) in the case of isotropic single-layer TMDs $\left(\mathrm{MoS}_{2}, \mathrm{MoSe}_{2}, \mathrm{WS}_{2}\right.$, and $\mathrm{WSe}_{2}$ ) the activity ratio of the $E^{\prime}$ to $A_{1}$ phonon mode, $\frac{I_{E^{\prime}}}{I_{A_{1}}}$, is a key to the determination of the induced strain since the ratio significantly increases when a tensile strain is applied while it decreases under compressive strain due to the opposite responses of the phonon modes, and (v) finally, a remarkable point for the anisotropic single-layers of $\operatorname{Re} X_{2}$ is that there is no significant change in Raman activities under biaxial strain.

In general, it was confirmed by the calculations that to extract strain information in single-layer 2D crystals, peak positions of lattice vibrational modes and the corresponding Raman activities are useful.

\section{ACKNOWLEDGMENTS}

Computational resources were provided by TUBITAK ULAKBIM, High Performance and Grid Computing Center (TR-Grid e-Infrastructure). H.S. acknowledges financial support from the Scientific and Technological Research Council of Turkey (TUBITAK) under Project No. 116C073. 
[1] K. S. Novoselov, A. K. Geim, S. V. Morozov, D. Jiang, Y. Zhang, S. V. Dubonos, I. V. Grigorieva, and A. A. Firsov, Science 306, 666 (2004).

[2] Q. H. Wang, K. Kalantar-Zadeh, A. Kis, J. N. Coleman, and M. S. Strano, Nat. Nanotechnol. 7, 699 (2012).

[3] G. R. Bhimanapati, Z. Lin, V. Meunier, Y. Jung, J. Cha, S. Das, D. Xiao, Y. Son, M. S. Strano, V. R. Cooper, L. Liang, S. G. Louie, E. Ringe, W. Zhou, S. S. Kim, R. R. Naik, B. G. Sumpter, H. Terrones, F. Xia, Y. Wang et al., ACS Nano. 9, 11509 (2015).

[4] D. Akinwande, N. Petrone, and J. Hone, Nat. Commun. 5, 5678 (2014).

[5] K. F. Mak, C. Lee, J. Hone, J. Shan, and T. F. Heinz, Phys. Rev. Lett. 105, 136805 (2010).

[6] R. A. Gordon, D. Yang, E. D. Crozier, D. T. Jiang, and R. F. Frindt, Phys. Rev. B 65, 125407 (2002).

[7] J. N. Coleman, M. Lotya, A. O’Neill, S. D. Bergin, P. J. King, U. Khan, K. Young, A. Gaucher, S. De, R. J. Smith, I. V. Shvets, S. K. Arora, G. Stanton, H. Y. Kim, K. Lee, G. T. Kim, G. S. Duesberg, T. Hallam, J. J. Boland, J. J. Wang et al., Science 331, 568 (2011).

[8] J. S. Ross, P. Klement, A. M. Jones, N. J. Ghimire, J. Yan, D. G. Mandrus, T. Taniguchi, K. Watanabe, K. Kitamura, W. Yao, D. H. Cobden, and X. Xu, Nat. Nanotechnol. 9, 268 (2014).

[9] H. Sahin, S. Tongay, S. Horzum, W. Fan, J. Zhou, J. Li, J. Wu, and F. M. Peeters, Phys. Rev. B 87, 165409 (2013).

[10] S. Tongay, H. Sahin, C. Ko, A. Luce, W. Fan, K. Liu, J. Zhou, Y. S. Huang, C. H. Ho, J. Yan, D. F. Ogletree, S. Aloni, J. Ji, S. Li, J. Li, F. M. Peeters, and J. Wu, Nat. Commun. 5, 3252 (2014).

[11] S. Horzum, D. Cakir, J. Suh, S. Tongay, Y. S. Huang, C. H. Ho, J. Wu, H. Sahin, and F. M. Peeters, Phys. Rev. B 89, 155433 (2014).

[12] B. Chen, H. Sahin, A. Suslu, L. Ding, M. I. Bertoni, F. M. Peeters, and S. Tongay, ACS Nano 9, 5326 (2015).

[13] A. Kara, H. Enriquez, A. P. Seitsonen, L. C. L. Y. Voon, S. Vizzini, B. Aufray, and H. Oughaddou, Surf. Sci. Rep. 67, 1 (2012).

[14] S. Cahangirov, M. Topsakal, E. Akturk, H. Sahin, and S. Ciraci, Phys. Rev. Lett. 102, 236804 (2009).

[15] H. Sahin, S. Cahangirov, M. Topsakal, E. Bekaroglu, E. Akturk, R. T. Senger, and S. Ciraci, Phys. Rev. B 80, 155453 (2009).

[16] Q. Wang, Q. Sun, P. Jena, and Y. Kawazoe, ACS Nano 3, 621 (2009).

[17] K. K. Kim, A. Hsu, X. Jia, S. M. Kim, Y. Shi, M. Hofmann, D. Nezich, J. F. Rodriguez-Nieva, M. Dresselhaus, T. Palacios, and J. Kong, Nano Lett. 12, 161 (2012).

[18] P. Tsipas, S. Kassavetis, D. Tsoutsou, E. Xenogiannopoulou, E. Golias, S. A. Giamini, C. Grazianetti, D. Chiappe, A. Molle, M. Fanciulli, and A. Dimoulas, Appl. Phys. Lett. 103, 251605 (2013).

[19] C. Bacaksiz, H. Sahin, H. D. Ozaydin, S. Horzum, R. T. Senger, and F. M. Peeters, Phys. Rev. B 91, 085430 (2015).

[20] D. Chenet, B. Aslan, P. Huang, C. Fan, A. van der Zande, T. Heinz, and J. Hone, Nano Lett. 15, 5667 (2015).

[21] L. Hart, S. Dale, S. Hoye, J. Webb, and D. Wolverson, Nano Lett. 16, 1381 (2016).

[22] M. Yagmurcukardes, C. Bacaksiz, R. T. Senger, and H. Sahin, 2D Mater. 4, 035013 (2017).

[23] S. Yang, S. Tongay, Y. Li, Q. Yue, J.-B. Xia, S.-S. Li, J. Li, and S.-H. Wei, Nanoscale 6, 7226 (2014).
[24] S. Yang, S. Tongay, Q. Yue, Y. Li, B. Li, and F. Lu, Sci. Rep. 4, 5442 (2014).

[25] J. Qiao, X. Kong, Z. X. Hu, F. Yang, and W. Ji, Nat. Commun. 5, 4475 (2014).

[26] F. Xia, H. Wang, and Y. Jia, Nat. Commun. 5, 4458 (2014).

[27] R. Fei and L. Yang, Nano Lett. 14, 2884 (2014).

[28] C. V. Raman, Indian J. Phys. 2, 387 (1928).

[29] Y. Okimoto, S. Horiuchi, E. Saitoh, R. Kumai, and Y. Tokura, Phys. Rev. Lett. 87, 187401 (2001).

[30] X.-F. Qiao, X.-L. Li, X. Zhang, W. Shi, J.-B. Wu, T. Chen, and P.-H. Tan, Appl. Phys. Lett. 106, 223102 (2015).

[31] X. Zhang, W. P. Han, J. B. Wu, S. Milana, Y. Lu, Q. Q. Li, A. C. Ferrari, and P. H. Tan, Phys. Rev. B 87, 115413 (2013).

[32] P. H. Tan, W. P. Han, W. J. Zhao, Z. H. Wu, K. Chang, H. Wang, Y. F. Wang, N. Bonini, N. Marzari, N. Pugno, G. Savini, A. Lombardo, and A. C. Ferrari, Nat. Mater. 11, 294 (2012).

[33] J.-B. Wu, X. Zhang, M. Ijas, W.-P. Han, X.-F. Qiao, X.-L. Li, D.-S. Jiang, A. C. Ferrari, and P.-H. Tan, Nat. Commun. 5, 5309 (2014).

[34] J.-B. Wu, Z.-X. Hu, X. Zhang, W.-P. Han, Y. Lu, W. Shi, X.-F. Qiao, M. Ijias, S. Milana, W. Ji, A. C. Ferrari, and P.-H. Tan, ACS Nano 9, 7440 (2015).

[35] Ph. Colomban, Spectrosc. Eur. 15, 8 (2003).

[36] G. Gouadec and Ph. Colomban, Prog. Cryst. Growth Charact. Mater. 53, 1 (2007).

[37] M. Havel, D. Baron, and Ph. Colomban, J. Mater. Sci. 39, 6183 (2004).

[38] X. Zhang, X.-F. Qiao, W. Shi, J.-B. Wu, D.-S. Jiang, and P.-H. Tan, Chem. Soc. Rev. 44, 2757 (2015).

[39] F. Guinea, M. I. Katsnelson, and A. K. Geim, Nat. Phys. 6, 30 (2009).

[40] K. He, C. Poole, K. F. Mak, and J. Shan, Nano Lett. 13, 2931 (2013).

[41] Z. H. Ni, T. Yu, Y. H. Lu, Y. Y. Wang, Y. P. Feng, and Z. X. Shen, ACS Nano 2, 2301 (2008).

[42] M. Huang, H. Yan, T. F. Heinz, and J. Hone, Nano Lett. 10, 4074 (2010).

[43] A. C. Ferrari and D. M. Basko, Nat. Nanotechnol. 8, 235 (2013).

[44] A. Berkdemir, H. R. Gutirrez, A. R. Botello-Méndez, N. PereaLópez, A. L. Elías, C.-I. Chia, B. Wang, V. H. Crespi, F. LópezUrías, J.-C. Charlier, H. Terrones, and M. Terronesa, Sci. Rep. 3, 1755 (2013).

[45] H. Frostig, T. Bayer, N. Dudovich, Y. C. Eldar, and Y. Silberberg, Nat. Photon. 9, 339 (2015).

[46] G. Kresse and J. Hafner, Phys. Rev. B 47, 558 (1993).

[47] G. Kresse and J. Furthmuller, Phys. Rev. B 54, 11169 (1996).

[48] J. P. Perdew, K. Burke, and M. Ernzerhof, Phys. Rev. Lett. 77, 3865 (1996).

[49] S. J. Grimme, J. Comput. Chem. 27, 1787 (2006).

[50] G. Henkelmana, A. Arnaldssonb, and H. Jónsson, Comput. Mater. Sci. 36, 354 (2006).

[51] M. Gmitra, S. Konschuh, C. Ertler, C. Ambrosch-Draxl, and J. Fabian, Phys. Rev. B 80, 235431 (2009).

[52] C. J. Tabert and E. J. Nicol, Phys. Rev. B 87, 235426 (2013).

[53] C.-C. Liu, W. Feng, and Y. Yao, Phys. Rev. Lett. 107, 076802 (2011).

[54] C.-C. Liu, H. Jiang, and Y. Yao, Phys. Rev. B 84, 195430 (2011). 
[55] K. K. Kim, A. Hsu, X. Jia, S. M. Kim, Y. Shi, M. Dresselhaus, T. Palacios, and J. Kong, ACS Nano 6, 8583 (2012).

[56] F. Huser, T. Olsen, and K. S. Thygesen, Phys. Rev. B 88, 245309 (2013).

[57] A. Ramasubramaniam, Phys. Rev. B 86, 115409 (2012).

[58] R. D’Souza and S. Mukherjee, Phys. Rev. B 95, 085435 (2017).

[59] G. Kern, G. Kresse, and J. Hafner, Phys. Rev. B 59, 8551 (1999).

[60] N. Dong, Y. Li, Y. Feng, S. Zhang, X. Zhang, C. Chang, J. Fan, L. Zhang, and J. Wang, Sci. Rep. 5, 14646 (2015).

[61] C. Rice, R. J. Young, R. Zan, U. Bangert, D. Wolverson, T. Georgiou, R. Jalil, and K. S. Novoselov, Phys. Rev. B 87, 081307 (2013).
[62] Y. Feng, W. Zhou, Y. Wang, J. Zhou, E. Liu, Y. Fu, Z. Ni, X. Wu, H. Yuan, F. Miao, B. Wang, X. Wan, and D. Xing, Phys. Rev. B 92, 054110 (2015).

[63] L. Li, Y. Yu, G. J. Ye, Q. Ge, X. Ou, H. Wu, D. Feng, X. H. Chen, and Y. Zhang, Nat. Nanotechnol. 9, 372 (2014).

[64] H. O. H. Churchill and P. J.-Herrero, Nat. Nanotechnol. 9, 330 (2014).

[65] X. Wang, A. M. Jones, K. L. Seyler, V. Tran, Y. Jia, H. Zhao, H. Wang, L. Yang, X. Xu, and F. Xia, Nat. Nanotechnol. 10, 517 (2015).

[66] A. Favron, E. Gaufres, F. Fossard, A.-L. P.- LHeureux, N. Y.-W. Tang, P. L. Levesque, A. Loiseau, R. Leonelli, S. Francoeur, and R. Martel, Nat. Mater. 14, 826 (2015). 